\title{
ON WELL-POSEDNESS FOR THE INHOMOGENEOUS NONLINEAR SCHRÖDINGER EQUATION IN THE CRITICAL CASE
}

\author{
JUNGKWON KIM, YOONJUNG LEE AND IHYEOK SEO
}

\begin{abstract}
In this paper we study the well-posedness for the inhomogeneous nonlinear Schrödinger equation $i \partial_{t} u+\Delta u=\lambda|x|^{-\alpha}|u|^{\beta} u$ in Sobolev spaces $H^{s}$, $s \geq 0$. The well-posedness theory for this model has been intensively studied in recent years, but much less is understood compared to the classical NLS model where $\alpha=0$. The conventional approach does not work particularly for the critical cases $\beta=\frac{4-2 \alpha}{d-2 s}$. It is still an open problem. The main contribution of this paper is to develop the well-posedness theory in this critical case (as well as non-critical cases). To this end, we approach to the matter in a new way based on a weighted $L^{p}$ setting which seems to be more suitable to perform a finer analysis for this model. This is because it makes it possible to handle the singularity $|x|^{-\alpha}$ in the nonlinearity more effectively. This observation is a core of our approach that covers the critical case successfully.
\end{abstract}

\section{INTRODUCTION}

In this paper we are concerned with the Cauchy problem for the inhomogeneous nonlinear Schrödinger equation (INLS)

$$
\left\{\begin{array}{l}
i \partial_{t} u+\Delta u=\lambda|x|^{-\alpha}|u|^{\beta} u, \quad(x, t) \in \mathbb{R}^{d} \times \mathbb{R}, \\
u(x, 0)=u_{0}(x)
\end{array}\right.
$$

where $\alpha, \beta>0$ and $\lambda= \pm 1$. The equation is called focusing INLS when $\lambda=-1$ and defocusing INLS when $\lambda=1$. This type of equation arises in nonlinear optics and plasma physics for the propagation of laser beams in an inhomogeneous medium ([2, 22]). The case $\alpha=0$ is the classical nonlinear Schrödinger equation (NLS) whose well-posedness theory in Sobolev spaces $H^{s}$ has been extensively studied over the past several decades and is well understood $([6,7,13,14,16,23])$. However, much less is known about the INLS equation which has drawn attention in recent years.

Before reviewing known results for the Cauchy problem (1.1), we recall the critical Sobolev index from which one can divide the matter into three cases. Note first that if $u(x, t)$ is a solution of (1.1) so is $u_{\lambda}(x, t)=\lambda^{\frac{2-\alpha}{\beta}} u\left(\lambda x, \lambda^{2} t\right)$, with the initial data $u_{\lambda, 0}(x)=u_{\lambda}(x, 0)$ for all $\lambda>0$. We then easily see

$$
\left\|u_{\lambda, 0}\right\|_{\dot{H}^{s}}=\lambda^{s+\frac{2-\alpha}{\beta}-\frac{d}{2}}\left\|u_{0}\right\|_{\dot{H}^{s}}
$$

2010 Mathematics Subject Classification. Primary: 35A01, 35Q55; Secondary: 35B45.

Key words and phrases. Well-posedness, nonlinear Schrödinger equations, weighted estimates.

This research was supported by NRF-2019R1F1A1061316. 
from which the critical Sobolev index is given by $s_{c}=d / 2-(2-\alpha) / \beta$ which determines the scale-invariant Sobolev space $\dot{H}^{s_{c}}$. In this regard, the case $s_{c}=0$ (alternatively $\beta=\frac{4-2 \alpha}{d}$ ) is referred to as the mass-critical (or $L^{2}$-critical). If $s_{c}=1$ (alternatively $\beta=\frac{4-2 \alpha}{d-2}$ ) the problem is called the energy-critical (or $H^{1}$-critical), and finally it is known as the mass-supercritical and energy-subcritical if $0<s_{c}<1$. Therefore, we restrict our attention to the cases where $0 \leq s_{c} \leq 1$. We also assume $d \geq 3$ to make the review shorter.

The well-posedness for the INLS equation (1.1) was first studied by Genoud-Stuart [11] in the sense of distribution. Using the abstract theory developed by Cazenave [4, they showed that the focusing INLS equation with $0<\alpha<2$ is locally well-posed in $H^{1}\left(\mathbb{R}^{d}\right)$ if $0<\beta<\frac{4-2 \alpha}{d-2}$. In this case, Genoud [10] and Farah [9] also showed how small should be the initial data to have global well-posedness, respectively, in the spirit of Weinstein [26] and Holmer-Roudenko [15] for the classical case $\alpha=0$.

In all of the previous results mentioned above, the solution has been constructed in the energy space $H^{1}$ smaller than $L^{2}$. By comparison, Guzmán [12] used the standard contraction mapping argument based on the known classical Strichartz estimates to establish the well-posedness for the INLS equation (1.1) in various Sobolev spaces $H^{s}, s \leq 1$. His result in $H^{1}$ was also improved by Dinh [8] extending the validity of $\alpha$ in low dimensions. More importantly, Guzmán showed that (1.1) with $0<\alpha<2$ is locally well-posed in $L^{2}$ larger than the energy space $H^{1}$ if $0<\beta<\frac{4-2 \alpha}{d}$. He also treated the local well-posedness in $H^{s}$ for $\max \left\{0, s_{c}\right\}<s \leq 1$ if $0<\beta<\frac{4-2 \alpha}{d-2 s}$. But here, the critical case $\beta=\frac{4-2 \alpha}{d-2 s}$ with $0 \leq s \leq 1$ and $d \geq 3$ is left unsolved. Unlike the NLS equation where $\alpha=0([6,7])$, the conventional approach does not work for this case. It is still an open problem.

The main contribution of this paper is to develop the well-posedness theory in this critical case as well as the non-critical case. To this end, we approach to the matter in a new way based on a weighted $L^{p}$ setting which does seem to be more suitable to perform a finer analysis for the INLS model. This is because it makes it possible to deal with the singularity $|x|^{-\alpha}$ in the nonlinearity more effectively. This observation is a core of our approach that covers the critical case successfully.

Before stating our results, we introduce the following weighted space-time norms

$$
\|f\|_{L_{t}^{q} L_{x}^{r}\left(|x|^{-r \gamma}\right)}=\left(\int_{\mathbb{R}^{\prime}}\left(\int_{\mathbb{R}^{d}}|x|^{-r \gamma}|f(x)|^{r} d x\right)^{\frac{q}{r}} d t\right)^{\frac{1}{q}}
$$

where $1 \leq r<\infty$ and $\gamma \geq 0$. Our first result is then the following local well-posedness for the INLS equation (1.1) in $L^{2}$ up to the $L^{2}$-critical case, $\beta=(4-2 \alpha) / d$.

Theorem 1.1. Let $d \geq 3,0<\alpha<2$ and $0<\beta \leq(4-2 \alpha) / d$. Then for $u_{0} \in L^{2}\left(\mathbb{R}^{d}\right)$ there exist $T>0$ and a unique solution $u \in C\left([0, T] ; L^{2}\right) \cap L^{q}\left([0, T] ; L^{r}\left(|x|^{-r \gamma}\right)\right)$ of the problem (1.1) for

$$
\max \left\{0, \frac{\alpha-1}{\beta+1}\right\}<\gamma<\min \left\{1, \frac{\alpha}{\beta+1}\right\}
$$


and $(q, r)$ satisfying

$$
\begin{aligned}
& \frac{2}{q}=d\left(\frac{1}{2}-\frac{1}{r}\right)+\gamma, \quad \frac{\gamma}{2}<\frac{1}{q} \leq \frac{1}{2} \\
& \frac{1}{2(\beta+1)}<\frac{1}{r}<\frac{d-2(\alpha-1)}{2 d(\beta+1)}+\frac{\gamma}{d}
\end{aligned}
$$

Furthermore, the continuous dependence on initial data holds.

As we shall see in the proof of the theorem, we can give a precise estimate for the life span of the solution according to the size of the initial data, $T \sim\left\|u_{0}\right\|_{L^{2}}^{-4 \beta /(4-2 \alpha-d \beta)}$, in the subcritical case. Thanks to the mass conservation for the INLS equation,

$$
\text { Mass } \equiv M[u(t)]=\int_{\mathbb{R}^{d}}|u(x, t)|^{2} d x=M\left[u_{0}\right],
$$

we can then apply the local result repeatedly, preserving the length of the time interval to get a global solution. However, the situation for the critical case is quite different. In this case, the local solution exists in a time interval depending on the data $u_{0}$ itself and not on its norm. Thus, the conservation (1.2) does not guarantee the existence of a global solution any more. For this reason, $\left\|u_{0}\right\|_{L^{2}}$ is assumed to be small for the critical case in the following global result.

Corollary 1.2. Under the same conditions as in Theorem 1.1, the local solution extends globally in time with $u \in C\left([0, \infty) ; L^{2}\right) \cap L^{q}\left([0, \infty) ; L^{r}\left(|x|^{-r \gamma}\right)\right)$ for $u_{0} \in$ $L^{2}\left(\mathbb{R}^{d}\right)$. Particularly in the critical case $\beta=(4-2 \alpha) / d,\left\|u_{0}\right\|_{L^{2}}$ is assumed to be small and the solution scatters in $L^{2}$, i.e., there exists $\varphi \in L^{2}$ such that

$$
\lim _{t \rightarrow \infty}\left\|u(t)-e^{i t \Delta} \varphi\right\|_{L_{x}^{2}}=0 \text {. }
$$

When $\alpha=0$, the INLS model becomes the classical NLS equation which has been the subject of intensive work for a long time. In this case, the $L^{2}$ theory was obtained by Tsutsumi [23] when $0<\beta<4 / d$. The $L^{2}$-critical case $\beta=4 / d$ was later treated by Cazenave-Weissler [6]. Particularly when $\alpha=0$ in our approach, it is deduced that $\gamma=0$ (see Remark 1.6), and in this case resulting results cover this classical results for the NLS equation.

We also obtain the following local well-posedness in $H^{s}$ for $s>0$ up to the critical case $\beta=(4-2 \alpha) /(d-2 s)$ which is equivalent to $s_{c}=s$.

Theorem 1.3. Let $d \geq 3$ and $0<s<1 / 3$. Assume that

$$
\max \left\{\frac{26-3 d}{12}, \frac{12 s+4 d s-8 s^{2}}{d+4 s}\right\}<\alpha<2
$$

and

$$
\max \left\{0, \frac{10 s-2 \alpha}{d-6 s}\right\}<\beta \leq \frac{4-2 \alpha}{d-2 s} .
$$

Then for $u_{0} \in H^{s}\left(\mathbb{R}^{d}\right)$ there exist $T>0$ and a unique solution $u \in C\left([0, T] ; H^{s}\right) \cap$ $L^{q}\left([0, T] ; L^{r}\left(|x|^{-r \gamma}\right)\right)$ of the problem (1.1) if

$$
\max \left\{3 s, \frac{\alpha+s-1}{\beta+1}\right\}<\gamma<\min \left\{1+s, \frac{\alpha-s}{\beta+1}, \frac{d \beta+2 \alpha-4 s}{2(\beta+1)}\right\}
$$


and $(q, r)$ satisfies

$$
\begin{gathered}
\frac{2}{q}=d\left(\frac{1}{2}-\frac{1}{r}\right)+\gamma-s, \quad \frac{\gamma-s}{2}<\frac{1}{q} \leq \frac{1}{2}, \\
\max \left\{\frac{1}{2(\beta+1)}, \frac{1}{2(\beta+1)}+\frac{2 s-\alpha}{d(\beta+1)}+\frac{\gamma}{d}\right\}<\frac{1}{r}<\frac{d-2 s-2(\alpha-1)}{2 d(\beta+1)}+\frac{\gamma}{d} .
\end{gathered}
$$

Furthermore, the continuous dependence on initial data holds.

Remark 1.4. When $\beta \leq(4-2 \alpha) / d$, we already have the well-posedness result in $L^{2}$ which implies automatically a result in $H^{s}$. Hence an essential part in the latter case is when $\beta>(4-2 \alpha) / d$. The range of $\beta$ in the theorem contains this range when $\alpha \geq 5 s$, and when $\alpha<5 s$ if we further assume $\alpha \geq \frac{5 d s-2 d+12 s}{6 s}$.

One of the most basic tools for the well-posedness of nonlinear dispersive equations is the contraction mapping principle. The key ingredient in this argument is the availability of Strichartz estimates. Indeed, Guzmán [12 makes use of the fractional product and chain rules to derive a contraction from the nonlinearity in (1.1) based on the classical Strichartz estimates. Unfortunately, this approach does not work for the critical case. In our approach we make use of the weighted Strichartz estimates (see (1.5) and (1.7) ) which deal with the singularity $|x|^{-\alpha}$ in the nonlinearity more effectively. More interestingly, we take advantage of the smoothing estimates (see (1.6) or (1.8) ) which makes it possible to apply more directly the contraction mapping principle without using the fractional product and chain rules. As consequences, we can handle the $H^{s}$-critical cases, and even $\left.u \in L_{t}^{q} L_{x}^{r}\left(|x|^{-r \gamma}\right)\right)$ in which $L_{x}^{r}$ is less restrictive than the usual $H_{x}^{s, r}$. Indeed, there are no less restrictive alternatives than $L_{x}^{r}$ within the framework of Strichartz estimates. These aspects are a core of our approach which establishes the well-posedness theory up to the critical case successfully.

Proposition 1.5. Let $d \geq 3$ and $0 \leq s<1 / 2$. Assume that $(q, r)$ and $(\tilde{q}, \tilde{r})$ satisfy

$$
\begin{aligned}
& \frac{2}{q}=d\left(\frac{1}{2}-\frac{1}{r}\right)+\gamma-s, \quad \frac{\gamma-s}{2}<\frac{1}{q} \leq \frac{1}{2}, \quad \frac{\gamma-s}{2} \leq \frac{1}{r}<\frac{1}{2}, \\
& \frac{2}{\tilde{q}}=d\left(\frac{1}{2}-\frac{1}{\tilde{r}}\right)+\tilde{\gamma}+s, \quad \frac{\tilde{\gamma}+s}{2}<\frac{1}{\tilde{q}} \leq \frac{1}{2}, \quad \frac{\tilde{\gamma}+s}{2} \leq \frac{1}{\tilde{r}}<\frac{1}{2},
\end{aligned}
$$

for $3 s<\gamma<1+s$ and $s<\tilde{\gamma}<1-s$. Then we have

$$
\begin{aligned}
& \left\|e^{i t \Delta} f\right\|_{L_{t}^{q} L_{x}^{r}\left(|x|^{-r \gamma}\right)} \lesssim\|f\|_{\dot{H}^{s}}, \\
& \left\||\nabla|^{s} \int_{-\infty}^{\infty} e^{-i \tau \Delta} F(\cdot, \tau) d \tau\right\|_{L^{2}} \lesssim\|F\|_{L_{t}^{\tilde{q}^{\prime}} L_{x}^{\tilde{r}^{\prime}}\left(|x|^{\tilde{r}^{\prime} \tilde{\gamma}}\right)},
\end{aligned}
$$

and if we additionally assume $q>\tilde{q}^{\prime}$

$$
\left\|\int_{0}^{t} e^{i(t-\tau) \Delta} F(\cdot, \tau) d \tau\right\|_{L_{t}^{q} L_{x}^{r}\left(|x|^{-r \gamma}\right)} \lesssim\|F\|_{L_{t}^{\tilde{q}^{\prime} L_{x}^{\tilde{r}^{\prime}}\left(|x|^{\tilde{r}^{\prime} \tilde{\gamma}}\right)}} .
$$


Remark 1.6. Particularly when $s=0$, the above three estimates also hold for the case where $\gamma=0$ and $\tilde{\gamma}=0$, including $(q, r)=(\infty, 2)$ and $(\tilde{q}, \tilde{r})=(\infty, 2)$. The first two estimates (1.5) and (1.6) also hold for $(q, r)=(2,2)$ with $\gamma=1+s$ and $(\tilde{q}, \tilde{r})=(2,2)$ with $\tilde{\gamma}=1-s$, respectively. Using these cases in our approach, one is led to have the theorems up to the boundary points in some ranges of $q, r, \gamma$. But this is an insignificant part, and hence we omit the details.

Remark 1.7. The first conditions in (1.3) and (1.4) are just the scaling conditions for which the estimates hold.

It should be also noted that (1.6) is equivalent to

$$
\left\||\nabla|^{s} e^{i t \Delta} f\right\|_{L_{t}^{\tilde{q}} L_{x}^{\tilde{r}}\left(|x|^{-\tilde{r} \tilde{\gamma}}\right)} \lesssim\|f\|_{L^{2}}
$$

by duality. This shows the smoothing effect of the Schrödinger propagator. To obtain the well-posedness in $H^{s}, s>0$, we make use of this type of smoothing estimates because it allows us to have the inhomogeneous estimate (1.7) without derivative from which it is possible to apply directly the contraction mapping principle to the nonlinearity in (1.1) without using the fractional product and chain rules. Indeed, by the standard $T T^{*}$ argument, (1.5) and (1.6) with the same $s$ implies (1.7) without derivative. In this regard, we need to have the common range of $s, 0 \leq s<1 / 2$, for which both (1.5) and (1.6) hold, although (1.5) holds more widely for $0 \leq s<(d-2) / 2$ (see Section 2 for details).

Outline of paper. In Section 2, we prove Proposition 1.5 by making use of the complex interpolation between the classical Strichartz estimates and the Kato-Yajima smoothing estimates appealing to the complex interpolation space identities. In Section 3 we obtain some weighted estimates for the nonlinear term $|x|^{-\alpha}|u|^{\beta} u$ of the INLS equation. These nonlinear estimates will play a crucial role in later sections 4 and 5 to obtain the well-posedness results by applying the contraction mapping argument to the nonlinearity along with the estimates in Proposition 1.5.

Throughout this paper, the letter $C$ stands for a positive constant which may be different at each occurrence. We also denote $A \lesssim B$ to mean $A \leq C B$ with unspecified constants $C>0$.

\section{Proof of Proposition 1.5}

In this section we prove the estimates in Proposition 1.5. Let $d \geq 3$. We then first recall the classical Strichartz estimate

$$
\left\|e^{i t \Delta} f\right\|_{L_{t}^{a} L_{x}^{b}} \lesssim\|f\|_{L^{2}}
$$

which holds if and only if $\frac{2}{a}=d\left(\frac{1}{2}-\frac{1}{b}\right)$ and $2 \leq a, b \leq \infty$. This estimate was first established by Strichartz [20] in the diagonal case $a=b$ and then extended to mixed space-time norms as above (see [14, 18]). It is also necessary for us to make use of the Kato-Yajima smoothing estimate

$$
\left\|\left.|\nabla|\right|^{\rho} e^{i t \Delta} f\right\|_{L_{t, x}^{2}\left(|x|^{-2(1-\rho)}\right)} \lesssim\|f\|_{L^{2}}
$$


which holds if and only if $-\frac{d-2}{2}<\rho<\frac{1}{2}$. This estimate was first discovered by Kato and Yajima [17] for $0 \leq \rho<\frac{1}{2}$ and Ben-Artzi and Klainerman [1] gave an alternate proof of this result. Since then, the full range was obtained by Sugimoto 21, although it was later shown by Vilela 24] that the range is indeed optimal (see also [25]).

Now we make use of the complex interpolation between these two estimates (2.1) and (2.2) by appealing to the following complex interpolation space identities (see [3]).

Lemma 2.1. Let $0<\theta<1,1 \leq p_{0}, p_{1}<\infty$ and $s_{0}, s_{1} \in \mathbb{R}$. Then the following identities hold:

- Given two complex Banach spaces $A_{0}, A_{1}$,

$$
\left(L^{p_{0}}\left(A_{0}\right), L^{p_{1}}\left(A_{1}\right)\right)_{[\theta]}=L^{p}\left(\left(A_{0}, A_{1}\right)_{[\theta]}\right),
$$

and

$$
\left(L^{p_{0}}\left(w_{0}\right), L^{p_{1}}\left(w_{1}\right)\right)_{[\theta]}=L^{p}(w),
$$

with $1 / p=(1-\theta) / p_{0}+\theta / p_{1}$ and $w=w_{0}^{p(1-\theta) / p_{0}} w_{1}^{p \theta / p_{1}}$.

$\bullet$

$$
\left(\dot{H}^{s_{0}}, \dot{H}^{s_{1}}\right)_{[\theta]}=\dot{H}^{s}
$$

with $s=(1-\theta) s_{0}+\theta s_{1}, s_{0} \neq s_{1}$.

Here, $(\cdot, \cdot)_{[\theta]}$ denotes the complex interpolation functor.

Using the complex interpolation between (2.1) and (2.2), we first see

$$
\left\|e^{i t \Delta} f\right\|_{\left(L_{t}^{a} L_{x}^{b}, L_{t}^{2} L_{x}^{2}\left(|x|^{-2(1-\rho)}\right)\right)_{[\theta]}} \lesssim\|f\|_{\left(\dot{H}^{0}, \dot{H}^{-\rho}\right)_{[\theta]}},
$$

and then we make use of Lemma 2.1 to get

$$
\left\|e^{i t \Delta} f\right\|_{L_{t}^{q} L_{x}^{r}\left(|x|^{-r \gamma}\right)} \lesssim\|f\|_{\dot{H}^{-\sigma}}
$$

where

$$
\frac{1}{q}=\frac{1-\theta}{a}+\frac{\theta}{2}, \quad \frac{1}{r}=\frac{1-\theta}{b}+\frac{\theta}{2}, \quad \gamma=(1-\rho) \theta, \quad \sigma=\rho \theta
$$

under the conditions

$$
2 \leq a, b \leq \infty,(a, b) \neq(\infty, 2), \frac{2}{a}=d\left(\frac{1}{2}-\frac{1}{b}\right), 0<\theta<1,-\frac{d-2}{2}<\rho<\frac{1}{2} .
$$

By eliminating the redundant exponents $a, b, \rho, \theta$ here, all the conditions on $q, r, \gamma, \sigma$ for which (2.3) holds are summarized as follows:

$$
\begin{gathered}
0<\gamma+\sigma<1, \quad-\frac{\gamma(d-2)}{d}<\sigma<\gamma, \\
\frac{2}{q}=d\left(\frac{1}{2}-\frac{1}{r}\right)+\gamma+\sigma, \quad \frac{\gamma+\sigma}{2} \leq \frac{1}{q}, \frac{1}{r} \leq \frac{1}{2}, \quad\left(\frac{1}{q}, \frac{1}{r}\right) \neq\left(\frac{\gamma+\sigma}{2}, \frac{1}{2}\right) .
\end{gathered}
$$

Indeed, from the last two conditions in (2.4) it follows that $\theta=\gamma+\sigma$. The third condition in (2.5) implies $2 / q=d(1 / 2-1 / r)+\theta$. Hence the first conditions in (2.6) and (2.7) are concluded. Combining the last conditions in (2.4) and (2.5), we see $-(d-2) \theta / 2<\sigma<\theta / 2$, and then substituting $\theta=\gamma+\sigma$ implies the second condition 
in (2.6). Finally, applying the first two conditions in (2.5) with $\theta=\gamma+\sigma$ to the first two ones in (2.4), the last two conditions in (2.7) are concluded.

Now let $s \geq 0$. By substituting $-s$ for $\sigma$ in (2.3), we obtain the first estimate (1.5) in the proposition because (2.7) implies directly the condition (1.3) and (2.6) implies $\frac{d}{d-2} s<\gamma<1+s$ which is wider than $3 s<\gamma<1+s$. On the other hand, we substitute $s, \tilde{q}, \tilde{r}, \tilde{\gamma}$ for $\sigma, q, r, \gamma$, respectively in (2.3) to obtain (1.8) which is equivalent to the second estimate (1.6) in the proposition. In this case, (2.7) implies directly the condition (1.4) and it is not difficult to see that (2.6) implies the condition $s<\tilde{\gamma}<1-s$. Hence the common range of $s$ for which both (1.5) and (1.6) hold is given by $0 \leq s<1 / 2$. This is the reason why we choose $3 s<\gamma<1+s$ instead of $\frac{d}{d-2} s<\gamma<1+s$ as the range of $\gamma$ for which (1.5) holds.

It remains to prove (1.7). By the $T T^{*}$ argument, (1.5) and (1.6) with the same value of $s$ imply

$$
\left\|\int_{-\infty}^{\infty} e^{i(t-\tau) \Delta} F(\cdot, \tau) d \tau\right\|_{L_{t}^{q} L_{x}^{r}\left(|x|^{-\gamma \gamma}\right)} \lesssim\|F\|_{L_{t}^{\tilde{q}^{\prime}} L_{x}^{\tilde{r}^{\prime}}\left(|x| \tilde{r}^{\prime} \tilde{\gamma}\right)} .
$$

By applying the following Christ-Kiselev lemma [5], we now get (1.7) for $q>\tilde{q}^{\prime}$ as desired.

Lemma 2.2. Let $X$ and $Y$ be two Banach spaces and let $T$ be a bounded linear operator from $L^{\alpha}(\mathbb{R} ; X)$ to $L^{\beta}(\mathbb{R} ; Y)$ such that

$$
T f(t)=\int_{\mathbb{R}} K(t, s) f(s) d s
$$

Then the operator

$$
\widetilde{T} f(t)=\int_{-\infty}^{t} K(t, s) f(s) d s
$$

has the same boundedness when $\beta>\alpha$, and $\|\widetilde{T}\| \lesssim\|T\|$.

In particular, if $s=0$, the estimate (1.5) for the case $\gamma=0$ is just the same as (2.1), and (1.6) for the case $\tilde{\gamma}=0$ follows from its dual estimate. By the $T T^{*}$ argument together with these cases, (1.7) follows for the case where $s=0$ and $\gamma, \tilde{\gamma}=0$. It follows also directly from (2.2) that (1.5) and (1.6) hold for $(q, r)=(2,2)$ with $\gamma=1+s$ and $(\tilde{q}, \tilde{r})=(2,2)$ with $\tilde{\gamma}=1-s$, respectively. This shows Remark1.6.

\section{Nonlinear ESTimates}

In this section we obtain some weighted estimates for the nonlinearity of the INLS equation using the same spaces as those involved in the weighted Strichartz estimates in Proposition 1.5. These nonlinear estimates will play a crucial role in later sections to obtain the well-posedness results applying the contraction mapping principle.

3.1. The $L^{2}$ case. We first establish the nonlinear estimates which are necessary for us to obtain the well-posedness in $L^{2}$ in the next section. 
Lemma 3.1. Let $d \geq 3,0<\alpha<2$ and $0<\beta \leq(4-2 \alpha) / d$. Assume that the exponents $q, r, \gamma$ satisfy all the conditions given as in Theorem 1.1. Then there exist certain $\tilde{q}, \tilde{r}, \tilde{\gamma}$ satisfying all the conditions given as in Proposition 1.5 with $s=0$ for which

$$
\left\||x|^{-\alpha}|u|^{\beta} v\right\|_{L_{t}^{\tilde{q}^{\prime}\left(I ; L_{x}^{\tilde{r}^{\prime}}\left(|x|^{\tilde{r}^{\prime} \tilde{\gamma}}\right)\right)}} \leq T^{\theta_{0}}\|u\|_{L_{t}^{q}\left(I ; L_{x}^{r}\left(|x|^{-r \gamma}\right)\right)}^{\beta}\|v\|_{L_{t}^{q}\left(I ; L_{x}^{r}\left(|x|^{-r \gamma}\right)\right)}
$$

holds for any finite interval $I=[0, T]$ and

$$
\theta_{0}=-\frac{d \beta}{4}+1-\frac{\alpha}{2}
$$

Remark 3.2. It should be noted that $\theta_{0} \geq 0$ if and only if $\beta \leq(4-2 \alpha) / d$.

Proof of Lemma 3.1. Let us first consider the exponent pairs $(q, r, \gamma)$ and $(\tilde{q}, \tilde{r}, \widetilde{\gamma})$ satisfying $q>\tilde{q}^{\prime}$,

$$
\begin{aligned}
& \frac{2}{q}=d\left(\frac{1}{2}-\frac{1}{r}\right)+\gamma, \quad \frac{\gamma}{2}<\frac{1}{q} \leq \frac{1}{2}, \quad \frac{\gamma}{2} \leq \frac{1}{r}<\frac{1}{2}, \quad 0<\gamma<1, \\
& \frac{2}{\tilde{q}}=d\left(\frac{1}{2}-\frac{1}{\tilde{r}}\right)+\tilde{\gamma}, \quad \frac{\tilde{\gamma}}{2}<\frac{1}{\tilde{q}} \leq \frac{1}{2}, \quad \frac{\tilde{\gamma}}{2} \leq \frac{1}{\tilde{r}}<\frac{1}{2}, \quad 0<\tilde{\gamma}<1,
\end{aligned}
$$

which are just the same given as in Proposition 1.5 with $s=0$ for which the estimates therein hold. We then let

$$
\frac{1}{\tilde{q}^{\prime}}=\theta_{0}+\frac{\beta+1}{q}, \quad \frac{1}{\tilde{r}^{\prime}}=\frac{\beta+1}{r} \text { and } \tilde{\gamma}-\alpha=-\gamma(\beta+1) .
$$

By combining the first conditions in (3.3) and (3.4) together with (3.5), it is not difficult to see that $\theta_{0}$ is determined by

$$
\theta_{0}=\frac{1}{\tilde{q}^{\prime}}-\frac{\beta+1}{q}=-\frac{d \beta}{4}+1-\frac{\alpha}{2}
$$

as in (3.2).

Next we use Hölder's inequality repeatedly along with (3.5) to get

$$
\begin{aligned}
& \left\||x|^{-\alpha}|u|^{\beta} v\right\|_{L_{t}^{\tilde{q}^{\prime}}\left(I ; L_{x}^{\tilde{r}^{\prime}}\left(|x| \tilde{r}^{\prime} \tilde{\gamma}\right)\right)}=\left\||x|^{\tilde{\gamma}-\alpha}|u|^{\beta} v\right\|_{L_{t}^{\tilde{q}^{\prime}\left(I ; L_{x}^{\tilde{r}^{\prime}}\right)}} \\
& =\left\||x|^{-\gamma(\beta+1)}|u|^{\beta} v\right\|_{L_{t}^{\tilde{q}^{\prime}}\left(I ; L_{x}^{\frac{r}{\beta+1}}\right)} \\
& \leq T^{\theta_{0}}\left\||x|^{-\gamma(\beta+1)}|u|^{\beta} v\right\|_{L_{t}^{\frac{q}{\beta+1}}\left(I ; L_{x}^{\frac{r}{\beta+1}}\right)} \\
& \leq T^{\theta_{0}}\|u\|_{L_{t}^{q}\left(I ; L_{x}^{r}\left(|x|^{-r \gamma}\right)\right)}^{\beta}\|v\|_{L_{t}^{q}\left(I ; L_{x}^{r}\left(|x|^{-r \gamma}\right)\right)}
\end{aligned}
$$

as desired in (3.1).

Now it remains to show the requirements on $q, r$ and $\gamma$ for which (3.1) holds. We first use the last two conditions in (3.5) to transform the exponents $\tilde{r}$ and $\tilde{\gamma}$ in (3.4) to $r$ and $\gamma$, as follows:

$$
\begin{gathered}
\frac{2}{\tilde{q}}=d\left(\frac{\beta+1}{r}-\frac{1}{2}\right)+\alpha-\gamma(\beta+1), \\
\frac{\alpha-\gamma(\beta+1)}{2}<\frac{1}{\tilde{q}} \leq \frac{1}{2}, \quad \frac{1}{2(\beta+1)}<\frac{1}{r} \leq \frac{2-\alpha}{2(\beta+1)}+\frac{\gamma}{2},
\end{gathered}
$$


and

$$
0<\alpha-\gamma(\beta+1)<1 .
$$

Next we substitute (3.6) into the first one in (3.7) to get

$$
\frac{1}{2(\beta+1)}<\frac{1}{r} \leq \frac{d-2(\alpha-1)}{2 d(\beta+1)}+\frac{\gamma}{d}
$$

Similarly we get

$$
\frac{d-2}{2 d}+\frac{\gamma}{d} \leq \frac{1}{r}<\frac{1}{2}
$$

by substituting the first one into the second one in (3.3). Notice here that the range of $1 / r$ in (3.3) may be replaced by (3.10) because $\frac{\gamma}{2} \leq \frac{d-2}{2 d}+\frac{\gamma}{d}$ when $\gamma<1$, and similarly using (3.8), it is easy to see that the second one in (3.7) may be replaced by (3.9). Finally, using the last two conditions in (3.5) and the first conditions in (3.3) and (3.4), one can easily see that $q>\tilde{q}^{\prime}$ is transformed to $\frac{1}{r}<\frac{2-\alpha}{d \beta}+\frac{\gamma}{d}$, but this may be replaced by (3.9). From the first one in (3.5), we also require $1 / \tilde{q}^{\prime}-1 / q=\theta_{0}+\beta / q>0$, but this is satisfied since $\frac{1}{q}>\frac{\gamma}{2}>0$.

Hence all the conditions on $q, r$ and $\gamma$ which we require are summarized as follows:

$$
\begin{aligned}
& \frac{2}{q}=d\left(\frac{1}{2}-\frac{1}{r}\right)+\gamma, \quad \frac{\gamma}{2}<\frac{1}{q} \leq \frac{1}{2}, \\
& \frac{1}{2(\beta+1)}<\frac{1}{r}<\frac{d-2(\alpha-1)}{2 d(\beta+1)}+\frac{\gamma}{d},
\end{aligned}
$$

and

$$
\max \left\{0, \frac{\alpha-1}{\beta+1}\right\}<\gamma<\min \left\{1, \frac{\alpha}{\beta+1}\right\}
$$

which are the same as in Theorem 1.1.

3.2. The $H^{s}$ case. Now we obtain some weighted estimates for the nonlinear term which will be used for the well-posedness in $H^{s}$ in Section 5 In comparison with the previous lemma, it is more delicate to obtain Lemma 3.3 below in which we need to get an additional estimate (3.14) having the term $|\nabla|^{-s}$ that is quite difficult to handle in the weighted spaces. For this, we will make use of a weighted version of the Sobolev embedding.

Lemma 3.3. Let $d \geq 3$ and $0<s<1 / 3$. Assume that

$$
\max \left\{\frac{26-3 d}{12}, \frac{12 s+4 d s-8 s^{2}}{d+4 s}\right\}<\alpha<2
$$

and

$$
\max \left\{0, \frac{10 s-2 \alpha}{d-6 s}\right\}<\beta \leq \frac{4-2 \alpha}{d-2 s} .
$$

If the exponents $q, r, \gamma$ satisfy all the conditions given as in Theorem 1.3, then there exist certain $\tilde{q}_{i}, \tilde{r}_{i}, \tilde{\gamma}_{i}, i=1,2$, satisfying all the conditions given as in Proposition 1.5 with $s>0$ for which

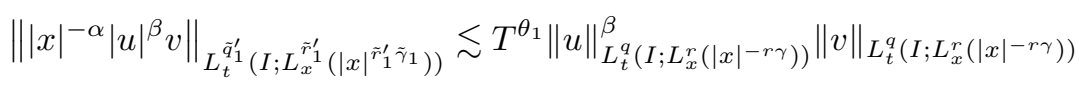


and

$$
\begin{aligned}
& \left\||\nabla|^{-s}\left(|x|^{-\alpha}|u|^{\beta} v\right)\right\|_{L_{t}^{\tilde{q}_{2}^{\prime}\left(I ; L_{x}^{\tilde{r}_{2}^{\prime}}\left(|x|^{\tilde{r}_{2}^{\prime}} \tilde{\gamma}_{2}\right)\right)}} \\
& \lesssim T^{\theta_{2}}\|u\|_{L_{t}^{q}\left(I ; L_{x}^{r}\left(|x|^{-r \gamma}\right)\right)}^{\beta}\|v\|_{L_{t}^{q}\left(I ; L_{x}^{r}\left(|x|^{-r \gamma}\right)\right)}
\end{aligned}
$$

hold for any finite interval $I=[0, T]$ and

$$
\theta_{1}=-\frac{d \beta}{4}+1-\frac{\alpha}{2}+\frac{s \beta}{2}, \quad \theta_{2}=-\frac{d \beta}{4}+1-\frac{\alpha}{2}+\frac{s(\beta+1)}{2} .
$$

Remark 3.4. We note that $\beta \leq(4-2 \alpha) /(d-2 s)$ if and only if $\theta_{1} \geq 0$ (or $\left.\theta_{2} \geq s / 2\right)$.

Proof of Lemma 3.3. First we consider the exponent pairs $(q, r, \gamma)$ and $\left(\tilde{q}_{i}, \tilde{r}_{i}, \tilde{\gamma}_{i}\right)$ for $i=1,2$ satisfying $q>\tilde{q}_{i}^{\prime}$,

$$
\begin{aligned}
& \frac{2}{q}=d\left(\frac{1}{2}-\frac{1}{r}\right)+\gamma-s, \quad \frac{\gamma-s}{2}<\frac{1}{q} \leq \frac{1}{2}, \quad \frac{\gamma-s}{2} \leq \frac{1}{r}<\frac{1}{2}, \quad 3 s<\gamma<1+s \\
& \frac{2}{\tilde{q}_{i}}=d\left(\frac{1}{2}-\frac{1}{\tilde{r}_{i}}\right)+\tilde{\gamma}_{i}+s, \frac{\tilde{\gamma}_{i}+s}{2}<\frac{1}{\tilde{q}_{i}} \leq \frac{1}{2}, \frac{\tilde{\gamma}_{i}+s}{2} \leq \frac{1}{\tilde{r}_{i}}<\frac{1}{2}, s<\tilde{\gamma}_{i}<1-s
\end{aligned}
$$

which are just the same given as in Proposition 1.5 with $0<s<1 / 2$ for which the estimates therein hold.

3.2.1. Proof of (3.13). The first estimate (3.13) is obtained in a similar way as in the previous subsection. Let us first set

$$
\frac{1}{\tilde{q}_{1}^{\prime}}=\theta_{1}+\frac{\beta+1}{q}, \quad \frac{1}{\tilde{r}_{1}^{\prime}}=\frac{\beta+1}{r} \quad \text { and } \quad \tilde{\gamma}_{1}-\alpha=-\gamma(\beta+1) .
$$

By combining the first conditions in (3.16) and (3.17) for $i=1$ together with (3.18), it is then easy to see that $\theta_{1}$ is determined by

$$
\theta_{1}=\frac{1}{\tilde{q}_{1}^{\prime}}-\frac{\beta+1}{q}=-\frac{d \beta}{4}+1-\frac{\alpha}{2}+\frac{s \beta}{2}
$$

as in (3.15).

Next we apply Hölder's inequality repeatedly along with (3.18) to get

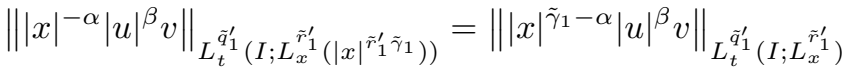

$$
\begin{aligned}
& =\left\||x|^{-\gamma(\beta+1)}|u|^{\beta} v\right\|_{L_{t}^{\tilde{q}_{1}^{\prime}\left(I ; L_{x}^{\frac{r}{\beta+1}}\right)}} \\
& \leq T^{\theta_{1}}\left\||x|^{-\gamma(\beta+1)}|u|^{\beta} v\right\|_{L_{t}^{\frac{q}{\beta+1}}\left(I ; L_{x}^{\frac{r}{\beta+1}}\right)} \\
& \leq T^{\theta_{1}}\|u\|_{L_{t}^{q}\left(I ; L_{x}^{r}\left(|x|^{-r \gamma}\right)\right)}^{\beta}\|v\|_{L_{t}^{q}\left(I ; L_{x}^{r}\left(|x|^{-r \gamma}\right)\right)}
\end{aligned}
$$

as desired in (3.13).

Now we only need to show the requirements on $q, r$ and $\gamma$ for which (3.13) holds. Using the last two conditions in (3.18), we first change the exponents $\tilde{r}_{1}$ and $\tilde{\gamma}_{1}$ in 
(3.17) for $i=1$ into $r$ and $\gamma$, as follows:

$$
\begin{gathered}
\frac{2}{\tilde{q}_{1}}=-\frac{d}{2}+\frac{d(\beta+1)}{r}+\alpha+s-\gamma(\beta+1), \\
\frac{\alpha-\gamma(\beta+1)+s}{2}<\frac{1}{\tilde{q}_{1}} \leq \frac{1}{2}, \quad \frac{1}{2(\beta+1)}<\frac{1}{r} \leq \frac{2-\alpha-s}{2(\beta+1)}+\frac{\gamma}{2} \\
\frac{\alpha+s-1}{\beta+1}<\gamma<\frac{\alpha-s}{\beta+1} .
\end{gathered}
$$

Next we insert (3.19) into the first one in (3.20) to get

$$
\frac{1}{2(\beta+1)}<\frac{1}{r} \leq \frac{1-\alpha-s}{d(\beta+1)}+\frac{1}{2(\beta+1)}+\frac{\gamma}{d}
$$

from which the second one in (3.20) can be removed using (3.21). Finally, all the requirements deduced from $q>\tilde{q}_{1}^{\prime}$ are already satisfied by the other requirements similarly as before.

In summary, the requirements on $q, r$ and $\gamma$ for which (3.13) holds are (3.16), (3.21) and (3.22) which are less restrictive than those in Theorem 1.3. But we will show that the common requirements for which both (3.13) and (3.14) hold are given exactly by those in Theorem 1.3 .

3.2.2. Proof of (3.14). Now we have to obtain (3.14) under the requirements (3.16), (3.21) and (3.22) on $q, r$ and $\gamma$ for which (3.13) holds. Let us first set

$$
\frac{1}{\tilde{q}_{2}^{\prime}}=\theta_{2}+\frac{\beta+1}{q}
$$

and then use Hölder's inequality in $t$ to the left-hand side of (3.14 to get

$$
\begin{aligned}
& \left\||\nabla|^{-s}\left(|x|^{-\alpha}|u|^{\beta} v\right)\right\|_{L_{t}^{\tilde{q}_{2}^{\prime}\left(I ; L_{x}^{\tilde{r}_{2}^{\prime}}\left(|x|^{\left.\left.\tilde{r}_{2}^{\prime} \tilde{\gamma}_{2}\right)\right)}\right.\right.}}=\left\||x|^{\tilde{\gamma}_{2}}|\nabla|^{-s}\left(|x|^{-\alpha}|u|^{\beta} v\right)\right\|_{L_{t}^{\tilde{q}_{2}^{\prime}\left(I ; L_{x}^{\tilde{r}_{2}^{\prime}}\right)}} \\
& \leq T^{\theta_{2}}\left\||x|^{\tilde{\gamma}_{2}}|\nabla|^{-s}\left(|x|^{-\alpha}|u|^{\beta} v\right)\right\|_{L_{t}^{\frac{q}{\beta+1}}\left(I ; L_{x}^{\tilde{r}_{2}^{\prime}}\right)} .
\end{aligned}
$$

To handle the term $|\nabla|^{-s}$ here, we make use of the following lemma which is a weighted version of the Sobolev embedding.

Lemma $3.5([19])$. Let $d \geq 1$ and $0<s<d$. If

$$
1<p \leq q<\infty, \quad-d / q<b \leq a<d / p^{\prime} \quad \text { and } \quad a-b-s=d / q-d / p,
$$

then

$$
\left\||x|^{b} f\right\|_{L^{q}} \leq C_{a, b, p, q}\left\||x|^{a}|\nabla|^{s} f\right\|_{L^{p}}
$$

Indeed, applying (3.24) with $a=\alpha-\gamma(\beta+1), b=\tilde{\gamma}_{2}, p=\frac{r}{\beta+1}, q=\tilde{r}_{2}^{\prime}$, and $f=|x|^{-\alpha}|u|^{\beta} v$, and then using Hölder's inequality, we get for $0<s<d$

$$
\begin{aligned}
\left\||x|^{\tilde{\gamma}_{2}}|\nabla|^{-s}\left(|x|^{-\alpha}|u|^{\beta} v\right)\right\|_{L_{t}^{\frac{q}{\beta+1}}\left(I ; L_{x}^{\tilde{r}_{2}^{\prime}}\right)} & \lesssim\left\||x|^{-\gamma(\beta+1)}|u|^{\beta} v\right\|_{L_{t}^{\frac{q}{\beta+1}}\left(I ; L_{x}^{\frac{r}{\beta+1}}\right)} \\
& \lesssim\left\||x|^{-\gamma} u\right\|_{L_{t}^{q}\left(I ; L_{x}^{r}\right)}^{\beta}\left\||x|^{-\gamma} v\right\|_{L_{t}^{q}\left(I ; L_{x}^{r}\right)}
\end{aligned}
$$


if

$$
\begin{gathered}
1<\frac{r}{\beta+1} \leq \tilde{r}_{2}^{\prime}<\infty \\
-\frac{d}{\tilde{r}_{2}^{\prime}}<\tilde{\gamma}_{2} \leq \alpha-\gamma(\beta+1)<d-\frac{d(\beta+1)}{r}
\end{gathered}
$$

and

$$
\tilde{\gamma}_{2}=\alpha-\gamma(\beta+1)-s-\frac{d}{\tilde{r}_{2}^{\prime}}+\frac{d(\beta+1)}{r} .
$$

Since $\tilde{\gamma}_{2}>0$, the first inequality in (3.26) is redundant, and since the upper bound of $1 / r$ in (3.22) is less than the one which follows from the third inequality in (3.26), it is also redundant. Hence (3.26) is reduced to

$$
\tilde{\gamma}_{2} \leq \alpha-\gamma(\beta+1) \text {. }
$$

Now we replace $\tilde{\gamma}_{2}$ in the first condition of (3.17) for $i=2$ with (3.27) to get

$$
\frac{2}{\tilde{q}_{2}}=-\frac{d}{2}+\alpha-\gamma(\beta+1)+\frac{d(\beta+1)}{r} .
$$

Then by inserting (3.29) and the first one of (3.16) into (3.23), we see that

$$
\theta_{2}=\frac{1}{\tilde{q}_{2}^{\prime}}-\frac{\beta+1}{q}=-\frac{d \beta}{4}+1-\frac{\alpha}{2}+\frac{s(\beta+1)}{2},
$$

as in (3.15).

By using (3.27) and (3.29), the exponents $\tilde{q}_{2}$ and $\tilde{\gamma}_{2}$ in all the inequalities in (3.17) for $i=2$ can be removed as follows:

$$
\begin{aligned}
& \frac{1}{r} \leq \frac{1-\alpha}{d(\beta+1)}+\frac{1}{2(\beta+1)}+\frac{\gamma}{d}, \quad \frac{1}{\tilde{r}_{2}}<\frac{1}{2}, \\
& \alpha-\gamma(\beta+1)-\frac{d}{\tilde{r}_{2}^{\prime}}+\frac{d(\beta+1)}{r} \leq \frac{2}{\tilde{r}_{2}}<1, \\
& 2 s<\alpha-\gamma(\beta+1)-\frac{d}{\tilde{r}_{2}^{\prime}}+\frac{d(\beta+1)}{r}<1 .
\end{aligned}
$$

Here, (3.30) and (3.31) come from the second and third ones in (3.17), respectively, while (3.32) is derived from the last one in (3.17). Similarly, (3.25) and (3.28) are transformed to

$$
0 \leq \frac{\beta+1}{r}-\frac{1}{\tilde{r}_{2}^{\prime}}<\frac{1}{\tilde{r}_{2}} \text { and } \frac{d(\beta+1)}{r}-\frac{d}{\tilde{r}_{2}^{\prime}} \leq s,
$$

respectively. It is immediate that the first inequality in (3.30) is redundant by (3.22). Since $\tilde{r}_{2}>2$, (3.31) and (3.32) are reduced to

$$
2 s<\alpha-\gamma(\beta+1)-\frac{d}{\tilde{r}_{2}^{\prime}}+\frac{d(\beta+1)}{r} \leq \frac{2}{\tilde{r}_{2}}
$$

which is possible for $s<1 / \tilde{r}_{2}$. If $s<1 / \tilde{r}_{2}$, (3.33) is then reduced to

$$
0 \leq \frac{\beta+1}{r}-\frac{1}{\tilde{r}_{2}^{\prime}} \leq \frac{s}{d}
$$


In summary, we are reduced to (3.34) and (3.35), which are equivalent to

$$
\frac{2 s-\alpha+\gamma(\beta+1)}{d}-\frac{\beta+1}{r}+1<\frac{1}{\tilde{r}_{2}} \leq \frac{d-\alpha+\gamma(\beta+1)}{d-2}-\frac{d(\beta+1)}{(d-2) r}
$$

and

$$
1-\frac{\beta+1}{r} \leq \frac{1}{\tilde{r}_{2}} \leq \frac{s}{d}+1-\frac{\beta+1}{r}
$$

respectively, under the condition

$$
s<\frac{1}{\tilde{r}_{2}}<\frac{1}{2} .
$$

Now, it suffices to check that there exists $\tilde{r}_{2}$ satisfying (3.36), (3.37) and (3.38) under the conditions (3.16), (3.21) and (3.22). To do so, we first make each lower bound of $1 / \tilde{r}_{2}$ in (3.36), (3.37) and (3.38) less than all the upper bounds in turn. Then we are reduced to

$$
\frac{1}{r}<\frac{1-s}{\beta+1}+\frac{2 s-\alpha}{d(\beta+1)}+\frac{\gamma}{d}
$$

and

$$
\frac{1}{2(\beta+1)}+\frac{2 s-\alpha}{d(\beta+1)}+\frac{\gamma}{d}<\frac{1}{r}
$$

Indeed, starting the process from the lower bound in (3.36), we arrive at (3.39), $s-\alpha+\gamma(\beta+1)<0$, and (3.40), but the second one is redundant by (3.21). Similarly from the lower bound in (3.37), we arrive at $\frac{1}{r} \leq \frac{2-\alpha}{2(\beta+1)}+\frac{\gamma}{2}, 0 \leq \frac{s}{d}$, and $\frac{1}{2(\beta+1)}<\frac{1}{r}$. But here the first and third ones are replaced by (3.22) under (3.21), and the second one is redundant. Finally from the lower bound in (3.38), we arrive at (3.39), $\frac{1}{r}<$ $\frac{1-s}{\beta+1}+\frac{s}{d(\beta+1)}$, and $s<1 / 2$, but the second one is replaced by (3.39) using (3.21) and the last one is redundant. Nextly, we note that (3.39) may be also eliminated by (3.22) using the fact that $d-2-2 s d+6 s=(d-2)(1-2 s)+2 s>0$, and we combine the first two conditions in (3.16) to conclude

$$
\frac{1}{2}-\frac{1-\gamma+s}{d} \leq \frac{1}{r}<\frac{1}{2}
$$

which replaces the third condition in (3.16) since $\gamma-s<1$. Finally, all the requirements deduced from $q>\tilde{q}_{2}^{\prime}$ are already satisfied by the other requirements similarly as before.

Hence, all the requirements on $r$ are (3.22), (3.41) and (3.40) for which both (3.13) and (3.14) hold. In summary,

$$
\max \left\{\frac{1}{2(\beta+1)}, \frac{d-2 s-2}{2 d}+\frac{\gamma}{d}\right\}<\frac{1}{r}<\min \left\{\frac{d-2 s-2(\alpha-1)}{2 d(\beta+1)}+\frac{\gamma}{d}, \frac{1}{2}\right\}
$$

and

$$
\frac{1}{2(\beta+1)}+\frac{2 s-\alpha}{d(\beta+1)}+\frac{\gamma}{d}<\frac{1}{r}
$$

which are the same as in Theorem 1.3. Here, omitting the equality in (3.41) is harmless. 
Next we check that there exist $r$ satisfying (3.42) and 3.43). For this, we make each lower bound of $1 / r$ in them less than all the upper bounds in (3.42) in turn. Then the restrictions on $\gamma$ and $s$ are deduced as

$$
\gamma<\frac{d \beta+2 \alpha-4 s}{2(\beta+1)}
$$

and $0<s<1 / 3$. In fact, staring the process from the first lower bound in (3.42), we arrive at $\alpha+s-1-\gamma(\beta+1)<0$ and $\beta>0$, but the former is redundant by (3.21) as well as $\beta>0$. Similarly from the second lower bound in (3.42), we arrive at $\beta<\frac{4-2 \alpha}{d-2-2 s}$ and $\gamma<1+s$ which are obviously redundant. Finally from the lower bound in (3.43), we arrive at $s<1 / 3$ and (3.44).

Hence all the requirements on $\gamma$ for which both (3.13) and (3.14) hold are $3 s<$ $\gamma<1+s$, (3.21) and (3.44). In summary,

$$
\max \left\{3 s, \frac{\alpha+s-1}{\beta+1}\right\}<\gamma<\min \left\{1+s, \frac{\alpha-s}{\beta+1}, \frac{d \beta+2 \alpha-4 s}{2(\beta+1)}\right\}
$$

as in Theorem 1.3

To guarantee $\gamma$ satisfying all the requirements in (3.45) under $0<s<1 / 3$, we make each lower bound of $\gamma$ in (3.45) less than all the upper bounds in turn. Then we are reduced to

$$
\beta<\frac{\alpha-4 s}{3 s} \text { and } \frac{10 s-2 \alpha}{d-6 s}<\beta .
$$

Indeed, starting from the first lower bound, we see $s<1 / 2$ and (3.46). Nextly, from the second lower bound, we have $\alpha-2<\beta+s \beta, s<1 / 2$ and $6 s-2<d \beta$ which are clearly redundant.

Now the first condition in (3.46) is satisfied if $\frac{4-2 \alpha}{d-2 s}<\frac{\alpha-4 s}{3 s}$, which is equivalent to

$$
\frac{12 s+4 d s-8 s^{2}}{d+4 s}<\alpha
$$

as in Theorem 1.3. Then we need to check that there exist $\alpha \in\left(\frac{12 s+4 d s-8 s^{2}}{d+4 s}, 2\right)$. This is possible if

$$
-4 s^{2}+2 s+2 d s<d
$$

holds for $0<s<1 / 3$. To show this, we consider a quadratic function $f_{1}(s)=$ $-4 s^{2}+2(1+d) s$ which is concave downward. Since $f_{1}(s)$ is an increasing function for $s \in\left(0, \frac{d+1}{4}\right)$ including $s \in\left(0, \frac{1}{3}\right)$, the inequality (3.47) follows clearly from the fact that $f_{1}\left(\frac{1}{3}\right)<d$. Next we check that there exist $\beta$ satisfying the second condition of (3.46) under $0<s<1 / 3$. For this, we need to show $\frac{10 s-2 \alpha}{d-6 s}<\frac{4-2 \alpha}{d-2 s}$ which is equivalent to

$$
-10 s^{2}+(5 d+12-4 \alpha) s<2 d .
$$

Similarly, we consider a quadratic function $f_{2}(s)=-10 s^{2}+(5 d+12-4 \alpha) s$. It is also concave downward and increases for $s<\frac{5 d+12-4 \alpha}{20}$. Since $\frac{1}{3}<\frac{5 d+12-4 \alpha}{20}$, we require $f_{2}\left(\frac{1}{3}\right)<2 d$ to show (3.48), which is equivalent to

$$
\frac{26-3 d}{12}<\alpha
$$


as in Theorem 1.3. Hence, $\alpha$ and $\beta$ are required as in (3.11) and (3.12). Since $\frac{d}{2}-\frac{2-\alpha}{\beta} \leq s$ (i.e., $\beta \leq \frac{4-2 \alpha}{d-2 s}$ ), we finally require

$$
\frac{d}{2}-\frac{2-\alpha}{\beta}<\frac{1}{3}
$$

which is equivalent to $\beta<\frac{12-6 \alpha}{3 d-2}$. But here, $\frac{4-2 \alpha}{d-2 s}<\frac{12-6 \alpha}{3 d-2}$ for $0<s<1 / 3$. No more requirement occurs. This completes the proof.

\section{The WELL-POSEDNESS IN $L^{2}$}

In this section we prove Theorem 1.1 and Corollary 1.2 by applying the contraction mapping principle along with the weighted Strichartz estimates in Proposition 1.5 with $s=0$. The nonlinear estimates in Lemma 3.1 play a key role in this step.

4.1. The subcritical case. By Duhamel's principle, we first write the solution of the Cauchy problem (1.1) as

$$
\Phi_{u_{0}}(u)=e^{i t \Delta} u_{0}-i \lambda \int_{0}^{t} e^{i(t-\tau) \Delta} F(u) d \tau
$$

where $F(u)=|\cdot|^{-\alpha}|u(\cdot, \tau)|^{\beta} u(\cdot, \tau)$. For appropriate values of $T, M>0$, we shall show that $\Phi$ defines a contraction map on

$$
\begin{aligned}
& X(T, M)=\left\{u \in C_{t}\left(I ; L_{x}^{2}\right) \cap L_{t}^{q}\left(I ; L_{x}^{r}\left(|x|^{-r \gamma}\right)\right):\right. \\
& \left.\sup _{t \in I}\|u\|_{L_{x}^{2}}+\|u\|_{L_{t}^{q}\left(I ; L_{x}^{r}\left(|x|^{-r \gamma}\right)\right)} \leq M\right\},
\end{aligned}
$$

equipped with the distance

$$
d(u, v)=\sup _{t \in I}\|u-v\|_{L_{x}^{2}}+\|u-v\|_{L_{t}^{q}\left(I ; L_{x}^{r}\left(|x|^{-r \gamma}\right)\right)} .
$$

Here, $I=[0, T]$ and $(q, r, \gamma)$ is given as in Theorem 1.1

For this, we first show that $\Phi$ is well defined on $X$. Namely, for $u \in X$

$$
\sup _{t \in I}\|\Phi(u)\|_{L_{x}^{2}}+\|\Phi(u)\|_{L_{t}^{q}\left(I ; L_{x}^{r}\left(|x|^{-r \gamma}\right)\right)} \leq M .
$$

Using (1.5) and (1.7) combined with (4.1), we obtain

$$
\|\Phi(u)\|_{L_{t}^{q}\left(I ; L_{x}^{r}\left(|x|^{-r \gamma}\right)\right)} \leq C\left\|u_{0}\right\|_{L^{2}}+C\left\||x|^{-\alpha}|u|^{\beta} u\right\|_{L_{t}^{\tilde{q}^{\prime}\left(I ; L_{x}^{\tilde{r}^{\prime}}\left(|x|^{\tilde{r}^{\prime} \tilde{\gamma}}\right)\right)}} .
$$

By applying Lemma 3.1, it follows then that

$$
\begin{aligned}
\|\Phi(u)\|_{L_{t}^{q}\left(I ; L_{x}^{r}\left(|x|^{-r \gamma}\right)\right)} & \leq C\left\|u_{0}\right\|_{L^{2}}+C T^{\theta_{0}}\|u\|_{L_{t}^{q}\left(I ; L_{x}^{r}\left(|x|^{-r \gamma}\right)\right)}^{\beta+1} \\
& \leq C\left\|u_{0}\right\|_{L^{2}}+C T^{\theta_{0}} M^{\beta+1} .
\end{aligned}
$$

On the other hand, applying Plancherel's theorem, (1.6) and (3.1) in turn, we see

$$
\begin{aligned}
\sup _{t \in I}\|\Phi(u)\|_{L_{x}^{2}} & \leq C\left\|u_{0}\right\|_{L^{2}}+C\left\|\int_{-\infty}^{\infty} e^{-i \tau \Delta} \chi_{[0, t]}(\tau) F(u) d \tau\right\|_{L_{x}^{2}} \\
& \leq C\left\|u_{0}\right\|_{L^{2}}+C\|F(u)\|_{L_{t}^{\tilde{q}^{\prime}}\left(I ; L_{x}^{\tilde{r}^{\prime}}\left(|x| \tilde{r}^{\prime} \tilde{\gamma}\right)\right)} \\
& \leq C\left\|u_{0}\right\|_{L^{2}}+C T^{\theta_{0}} M^{\beta+1} .
\end{aligned}
$$


Hence, if we fix $M=4 C\left\|u_{0}\right\|_{L^{2}}$ and take $T>0$ such that

$$
C T^{\theta_{0}} M^{\beta} \leq \frac{1}{8}
$$

we get (4.2). In the subcritical case where $\beta<(4-2 \alpha) / d, T$ carries a positive power $\theta_{0}$ here. Thus one can give a precise estimate for the life span of the solution according to the size of the initial data, $T \sim\left\|u_{0}\right\|_{L^{2}}^{-\beta / \theta_{0}}$.

Next we show that $\Phi$ is a contraction. Namely, for $u, v \in X$

$$
d(\Phi(u), \Phi(v)) \leq \frac{1}{2} d(u, v) .
$$

Using the same arguments used in (4.4), we see

$$
\sup _{t \in I}\|\Phi(u)-\Phi(v)\|_{L_{x}^{2}} \leq C\|F(u)-F(v)\|_{L_{t}^{\tilde{q}^{\prime}}\left(I ; L_{x}^{\tilde{r}^{\prime}}\left(|x|^{\left.\left.\tilde{r}^{\prime} \tilde{\gamma}\right)\right)}\right.\right.} .
$$

Then we will show

$$
\|F(u)-F(v)\|_{L_{t}^{\tilde{q}^{\prime}}\left(I ; L_{x}^{\tilde{r}^{\prime}}\left(|x| \tilde{r}^{\prime} \tilde{\gamma}\right)\right)} \leq \frac{1}{4 C}\|u-v\|_{L_{t}^{q}\left(I ; L_{x}^{r}\left(|x|^{-r \gamma}\right)\right)} .
$$

Indeed, using the following simple inequality

$$
\left.|| u\right|^{\beta} u-|v|^{\beta} v \mid \leq C\left(|u|^{\beta}|u-v|+|v|^{\beta}|u-v|\right),
$$

we are reduced to showing

$$
\left\||x|^{-\alpha}|u|^{\beta}|u-v|\right\|_{L_{t}^{\tilde{q}^{\prime}\left(I ; L_{x}^{\tilde{r}^{\prime}}\left(|x|^{\tilde{r}^{\prime}} \tilde{\gamma}\right)\right)}} \leq \frac{1}{8 C}\|u-v\|_{L_{t}^{q}\left(I ; L_{x}^{r}\left(|x|^{-r \gamma}\right)\right)}
$$

by symmetry. For this we apply Lemma 3.1 with $v$ replaced by $|u-v|$ so that

$$
\begin{aligned}
\left\||x|^{-\alpha}|u|^{\beta}|u-v|\right\|_{L_{t}^{\tilde{q}^{\prime}\left(I ; L_{x}^{\tilde{r}^{\prime}}\left(|x| \tilde{r}^{\prime} \tilde{\gamma}\right)\right)}} & \leq T^{\theta_{0}}\|u\|_{L_{t}^{q}\left(I ; L_{x}^{r}\left(|x|^{-r \gamma}\right)\right)}^{\beta}\|u-v\|_{L_{t}^{q}\left(I ; L_{x}^{r}\left(|x|^{-r \gamma}\right)\right)} \\
& \leq T^{\theta_{0}} M^{\beta}\|u-v\|_{L_{t}^{q}\left(I ; L_{x}^{r}\left(|x|^{-r \gamma}\right)\right)},
\end{aligned}
$$

which implies (4.10) because we have chosen $T, M>0$ in (4.5) so that $T^{\theta_{0}} M^{\beta} \leq$ $1 /(8 C)$. On the other hand,

$$
\|\Phi(u)-\Phi(v)\|_{L_{t}^{q}\left(I ; L_{x}^{r}\left(|x|^{-r \gamma}\right)\right)} \leq C\|F(u)-F(v)\|_{L_{t}^{\tilde{q}^{\prime}}\left(I ; L_{x}^{\tilde{r}^{\prime}}\left(|x|^{\tilde{r}^{\prime} \tilde{\gamma}}\right)\right)} .
$$

Now we obtain (4.6) combining this, (4.7) and (4.8).

Therefore, we have proved that there exists a unique local solution

$$
u \in C\left(I ; L^{2}\right) \cap L^{q}\left(I ; L^{r}\left(|x|^{-r \gamma}\right)\right)
$$

with $T \sim\left\|u_{0}\right\|_{L^{2}}^{-\beta / \theta_{0}}$. The continuous dependence of the solution $u$ with respect to the initial data $u_{0}$ follows clearly in the same way:

$$
\begin{aligned}
d(u, v) & \lesssim d\left(e^{i t \Delta} u_{0}, e^{i t \Delta} v_{0}\right)+d\left(\int_{0}^{t} e^{i(t-\tau) \Delta} F(u) d \tau, \int_{0}^{t} e^{i(t-\tau) \Delta} F(v) d \tau\right) \\
& \lesssim\left\|u_{0}-v_{0}\right\|_{L^{2}} .
\end{aligned}
$$

Here, $u, v$ are the corresponding solutions for initial data $u_{0}, v_{0}$, respectively. Thanks to the mass conservation (1.2), the above process can be also iterated on translated time intervals, preserving the length of the time interval comparable to $\left\|u_{0}\right\|_{L^{2}}^{-\beta / \theta_{0}}$ to extend the above local solution globally in time. The proof is now complete. 
4.2. The critical case. The critical case requires slightly different arguments and it yields different conclusions. This is due to the fact that the power $\theta_{0}$ in the above argument becomes zero in this case. This time we cannot gain a small power of $T$ and the smallness must have a different source. For this reason, the global result will follow from the smallness of the initial data. We give the main lines of the proof.

We start from showing that $\Phi$ defines a contraction on

$$
\begin{aligned}
\widetilde{X}(T, M, N)=\{u & \in C_{t}\left(I ; L_{x}^{2}\right) \cap L_{t}^{q}\left(I ; L_{x}^{r}\left(|x|^{-r \gamma}\right)\right): \\
& \left.\sup _{t \in I}\|u\|_{L_{x}^{2}} \leq N, \quad\|u\|_{L_{t}^{q}\left(I ; L_{x}^{r}\left(|x|^{-r \gamma}\right)\right)} \leq M\right\}
\end{aligned}
$$

equipped with the distance

$$
d(u, v)=\sup _{t \in I}\|u-v\|_{L_{x}^{2}}+\|u-v\|_{L_{t}^{q}\left(I ; L_{x}^{r}\left(|x|^{-r \gamma}\right)\right)} .
$$

First, we see as in (4.4) and (4.3) that

$$
\sup _{t \in I}\|\Phi(u)\|_{L_{x}^{2}} \leq C\left\|u_{0}\right\|_{L^{2}}+C M^{\beta+1}
$$

and

$$
\|\Phi(u)\|_{L_{t}^{q}\left(I ; L_{x}^{r}\left(|x|^{-r \gamma}\right)\right)} \leq\left\|e^{i t \Delta} u_{0}\right\|_{L_{t}^{q}\left(I ; L_{x}^{r}\left(|x|^{-r \gamma}\right)\right)}+C M^{\beta+1},
$$

respectively. Observe here that

$$
\left\|e^{i t \Delta} u_{0}\right\|_{L_{t}^{q}\left(I ; L_{x}^{r}\left(|x|^{-r \gamma}\right)\right)} \leq \varepsilon
$$

for some $\varepsilon>0$ small enough which will be chosen later, provided that either $\left\|u_{0}\right\|_{L^{2}}$ is small (see (1.5) with $s=0$ ) or it is satisfied for some $T>0$ small enough by the dominated convergence theorem. Hence, one can take $T=\infty$ in the first case and $T$ to be this small time in the second. We therefore get $\Phi(u) \in \widetilde{X}$ for $u \in \widetilde{X}$ if

$$
C\left\|u_{0}\right\|_{L^{2}}+C M^{\beta+1} \leq N \text { and } \varepsilon+C M^{\beta+1} \leq M .
$$

On the other hand, using the same argument employed to show (4.6), we see

$$
\begin{aligned}
d(\Phi(u), \Phi(v)) & =\sup _{t \in I}\|\Phi(u)-\Phi(v)\|_{L_{x}^{2}}+\|\Phi(u)-\Phi(v)\|_{L_{t}^{q}\left(I ; L_{x}^{r}\left(|x|^{-r \gamma}\right)\right)} \\
& \leq C M^{\beta}\|u-v\|_{L_{t}^{q}\left(I ; L_{x}^{r}\left(|x|^{-r \gamma}\right)\right)} \\
& \leq C M^{\beta} d(u, v) .
\end{aligned}
$$

Now by setting $N=2 C\left\|u_{0}\right\|_{L^{2}}$ and $M=2 \varepsilon$ and then choosing $\varepsilon>0$ small enough so that (4.11) holds and $C M^{\beta} \leq 1 / 2$, it follows that $\widetilde{X}$ is stable by $\Phi$ and $\Phi$ is a contraction on $\widetilde{X}$.

Therefore, there exists a unique local solution $u \in C\left(I ; L^{2}\right) \cap L^{q}\left(I ; L^{r}\left(|x|^{-r \gamma}\right)\right)$ in the time interval $[0, T]$ with a small $T$. Recall from the above argument that when $\left\|u_{0}\right\|_{L^{2}}$ is small enough, we can take $T=\infty$ to obtain a global solution. The continuous dependence on the initial data $u_{0}$ follows clearly in the same way as before. 
It remains to prove the scattering property. Following the argument above, one can easily see that

$$
\begin{aligned}
& \left\|e^{-i t_{2} \Delta} u\left(t_{2}\right)-e^{-i t_{1} \Delta} u\left(t_{1}\right)\right\|_{L_{x}^{2}}=\left\|\int_{t_{1}}^{t_{2}} e^{-i \tau \Delta} F(u) d \tau\right\|_{L_{x}^{2}} \\
& \lesssim\|F(u)\|_{L_{t}^{\tilde{q}^{\prime}}\left(\left[t_{1}, t_{2}\right] ; L_{x}^{\tilde{r}^{\prime}}\left(|x| \tilde{r}^{\prime} \tilde{\gamma}\right)\right)} \\
& \lesssim\|u\|_{L_{t}^{q}\left(\left[t_{1}, t_{2}\right] ; L_{x}^{r}\left(|x|^{-r \gamma}\right)\right)}^{\beta+1} \quad \rightarrow \quad 0
\end{aligned}
$$

as $t_{1}, t_{2} \rightarrow \infty$. This implies that

$$
\varphi:=\lim _{t \rightarrow \infty} e^{-i t \Delta} u(t)
$$

exists in $L^{2}$. Furthermore, one has

$$
u(t)-e^{i t \Delta} \varphi=i \lambda \int_{t}^{\infty} e^{i(t-\tau) \Delta} F(u) d \tau,
$$

and hence

$$
\begin{aligned}
\left\|u(t)-e^{i t \Delta} \varphi\right\|_{L_{x}^{2}}=\left\|\int_{t}^{\infty} e^{i(t-\tau) \Delta} F(u) d \tau\right\|_{L_{x}^{2}} & \lesssim\|F(u)\|_{L_{t}^{\tilde{q}^{\prime}}\left([t, \infty) ; L_{x}^{\tilde{r}^{\prime}}\left(|x|^{\tilde{r}^{\prime} \tilde{\gamma}}\right)\right)} \\
& \lesssim\|u\|_{L_{t}^{q}\left([t, \infty) ; L_{x}^{r}\left(|x|^{-r \gamma}\right)\right)}^{\beta+1} \rightarrow 0
\end{aligned}
$$

as $t \rightarrow \infty$. This completes the proof.

\section{THE WELL-POSEDNESS IN $H^{s}$}

This final section is devoted to the proof of Theorem 1.3. The proof based on Proposition 1.5 and Lemma 3.3 is similar to the one given in the previous section for the $L^{2}$ case; therefore, we shall give only a sketch of it.

5.1. The subcritical case. For appropriate values of $T, M>0$, we show that $\Phi$ defines a contraction map on

$$
\begin{gathered}
X(T, M)=\left\{u \in C_{t}\left(I ; H_{x}^{s}\right) \cap L_{t}^{q}\left(I ; L_{x}^{r}\left(|x|^{-r \gamma}\right)\right):\right. \\
\left.\sup _{t \in I}\|u\|_{H_{x}^{s}}+\|u\|_{L_{t}^{q}\left(I ; L_{x}^{r}\left(|x|^{-r \gamma}\right)\right)} \leq M\right\}
\end{gathered}
$$

equipped with the distance

$$
d(u, v)=\sup _{t \in I}\|u-v\|_{H_{x}^{s}}+\|u-v\|_{L_{t}^{q}\left(I ; L_{x}^{r}\left(|x|^{-r \gamma}\right)\right)} .
$$

Here, $I=[0, T]$ and the exponent pair $(q, r, \gamma)$ is given as in Theorem 1.3. Firstly, $\Phi(u) \in X$ for $u \in X$, i.e.,

$$
\sup _{t \in I}\|\Phi(u)\|_{H_{x}^{s}}+\|\Phi(u)\|_{L_{t}^{q}\left(I ; L_{x}^{r}\left(|x|^{-r \gamma}\right)\right)} \leq M .
$$


Indeed, using (1.7) with $(\tilde{q}, \tilde{r}, \tilde{\gamma})=\left(\tilde{q}_{1}, \tilde{r}_{1}, \tilde{\gamma}_{1}\right)$ followed by (3.13) in Lemma 3.3 and (1.5), we see that

$$
\begin{aligned}
& \|\Phi(u)\|_{L_{t}^{q}\left(I ; L_{x}^{r}\left(|x|^{-r \gamma}\right)\right)} \leq C\left\|u_{0}\right\|_{H^{s}}+C\left\||x|^{-\alpha}|u|^{\beta} u\right\|_{L_{t}^{\tilde{q}_{1}^{\prime}}\left(I ; L_{x}^{\tilde{r}_{1}^{\prime}}\left(|x|^{\tilde{r}_{1}^{\prime} \tilde{\gamma}_{1}}\right)\right)} \\
& \leq C\left\|u_{0}\right\|_{H^{s}}+C T^{\theta_{1}}\|u\|_{L_{t}^{q}\left(I ; L_{x}^{r}\left(|x|^{-r \gamma}\right)\right)}^{\beta+1} \\
& \leq C\left\|u_{0}\right\|_{H^{s}}+C T^{\theta_{1}} M^{\beta+1}
\end{aligned}
$$

because $\|f\|_{\dot{H}^{s}} \lesssim\|f\|_{H^{s}}$. On the other hand, from Plancherel's Theorem combined with the smoothing estimate (1.6), we see

$$
\begin{aligned}
& \sup _{t \in I}\|u\|_{H_{x}^{s}} \leq C\left\|u_{0}\right\|_{H^{s}}+C \sup _{t \in I}\left\|\int_{0}^{t} e^{-i \tau \Delta} F(u) d \tau\right\|_{H^{s}}
\end{aligned}
$$

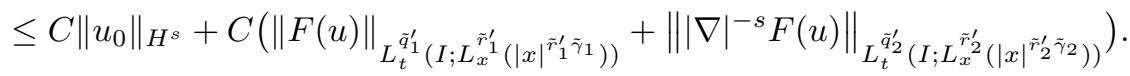

Here we also used, for the second inequality, that $\|f\|_{H^{s}} \lesssim\|f\|_{H^{s}}+\|f\|_{L^{2}}$. By using the nonlinear estimates (3.13) and (3.14), it follows then that

$$
\begin{aligned}
\sup _{t \in I}\|u\|_{H_{x}^{s}} & \leq C\left\|u_{0}\right\|_{H^{s}}+C\left(T^{\theta_{1}}+T^{\theta_{2}}\right)\|u\|_{L_{t}^{q}\left(I ; L_{x}^{r}\left(|x|^{-r \gamma}\right)\right)}^{\beta+1} \\
& \leq C\left\|u_{0}\right\|_{H^{s}}+C\left(T^{\theta_{1}}+T^{\theta_{2}}\right) M^{\beta+1} .
\end{aligned}
$$

Thus, if we set $M=4 C\left\|u_{0}\right\|_{H^{s}}$ and take $T>0$ such that

$$
C\left(T^{\theta_{1}}+T^{\theta_{2}}\right) M^{\beta} \leq \frac{1}{8}
$$

we obtain (5.1). Nextly, $\Phi$ is a contraction on $X$, i.e., for $u, v \in X$

$$
d(\Phi(u), \Phi(v)) \leq \frac{1}{2} d(u, v) .
$$

Indeed, we first see as above that

$$
\begin{aligned}
\sup _{t \in I}\|\Phi(u)-\Phi(v)\|_{H_{x}^{s}} \leq & C\|F(u)-F(v)\|_{L_{t}^{\tilde{q}_{1}^{\prime}}\left(I ; L_{x}^{\tilde{r}_{1}^{\prime}}\left(\mid x \tilde{r}_{1}^{\tilde{r}_{1}^{\prime} \tilde{\gamma}_{1}}\right)\right)} \\
& +C\left\||\nabla|^{-s}(F(u)-F(v))\right\|_{L_{t}^{\tilde{q}_{2}^{\prime}}\left(I ; L_{x}^{\tilde{r}_{2}^{\prime}}\left(|x|^{\tilde{r}_{2}^{\prime} \tilde{\gamma}_{2}}\right)\right)} .
\end{aligned}
$$

Using the simple inequality (4.9), the right-hand side here is bounded as in Lemma 3.3 by

$$
2 C\left(T^{\theta_{1}}+T^{\theta_{2}}\right) M^{\beta}\|u-v\|_{L_{t}^{q}\left(I ; L_{x}^{r}\left(|x|^{-r \gamma}\right)\right)} .
$$

Meanwhile, by (1.7) we have

$$
\|\Phi(u)-\Phi(v)\|_{L_{t}^{q}\left(I ; L_{x}^{r}\left(|x|^{-r \gamma}\right)\right)} \leq C\|F(u)-F(v)\|_{L_{t}^{\tilde{q}_{1}^{\prime}}\left(I ; L_{x}^{\tilde{r}_{1}^{\prime}}\left(|x|^{\tilde{r}_{1}^{\prime} \tilde{\gamma}_{1}}\right)\right)} .
$$

Consequently, we obtain (5.5) because of (5.4).

We have proved that there exists a unique solution $u \in C\left(I ; H^{s}\right) \cap L^{q}\left(I ; L^{r}\left(|x|^{-r \gamma}\right)\right)$ with $T=T\left(\left\|u_{0}\right\|_{H^{s}}, \alpha, \beta\right)$. The continuous dependence on the data follows clearly as before. 
5.2. The critical case. The situation in this case is similar to the $L^{2}$ case because the power $\theta_{1}$ in the above argument becomes zero in this case. But the other power $\theta_{2}=s / 2>0$ yields a slight different conclusion as shown in the proof.

We start from showing that $\Phi$ defines a contraction on

$$
\begin{aligned}
\tilde{X}(T, M, N)=\{u & \in C_{t}\left(I ; H^{s}\right) \cap L_{t}^{q}\left(I ; L_{x}^{r}\left(|x|^{-r \gamma}\right)\right): \\
& \left.\sup _{t \in I}\|u\|_{H_{x}^{s}} \leq N, \quad\|u\|_{L_{t}^{q}\left(I ; L_{x}^{r}\left(|x|^{-r \gamma}\right)\right)} \leq M\right\}
\end{aligned}
$$

equipped with the distance

$$
d(u, v)=\sup _{t \in I}\|u-v\|_{H_{x}^{s}}+\|u-v\|_{L_{t}^{q}\left(I ; L_{x}^{r}\left(|x|^{-r \gamma}\right)\right)} .
$$

As in (5.3) and (5.2), we see that

$$
\sup _{t \in I}\|\Phi(u)\|_{H_{x}^{s}} \leq C\left\|u_{0}\right\|_{H^{s}}+C\left(1+T^{\theta_{2}}\right) M^{\beta+1}
$$

and

$$
\|\Phi(u)\|_{L_{t}^{q}\left(I ; L_{x}^{r}\left(|x|^{-r \gamma}\right)\right)} \leq\left\|e^{i t \Delta} u_{0}\right\|_{L_{t}^{q}\left(I ; L_{x}^{r}\left(|x|^{-r \gamma}\right)\right)}+C M^{\beta+1},
$$

respectively. By the dominated convergence theorem we observe that

$$
\left\|e^{i t \Delta} u_{0}\right\|_{L_{t}^{q}\left(I ; L_{x}^{r}\left(|x|^{-r \gamma}\right)\right)} \leq \varepsilon
$$

for given $\varepsilon>0$ which will be chosen later, if $T>0$ is small enough. Hence, $\Phi(u) \in \widetilde{X}$ for $u \in \tilde{X}$ if

$$
C\left\|u_{0}\right\|_{H^{s}}+C\left(1+T^{\theta_{2}}\right) M^{\beta+1} \leq N \quad \text { and } \quad \varepsilon+C M^{\beta+1} \leq M
$$

Meanwhile, using the same argument employed to show (5.5), we see

$$
\begin{aligned}
d(\Phi(u), \Phi(v)) & =\sup _{t \in I}\|\Phi(u)-\Phi(v)\|_{H_{x}^{s}}+\|\Phi(u)-\Phi(v)\|_{L_{t}^{q}\left(I ; L_{x}^{r}\left(|x|^{-r \gamma}\right)\right)} \\
& \leq 2 C\left(1+T^{\theta_{2}}\right) M^{\beta} d(u, v) .
\end{aligned}
$$

Now we take $N=2 C\left\|u_{0}\right\|_{H^{s}}$ and $M=2 \varepsilon$, and then choose $\varepsilon>0$ small enough so that (5.7) holds and $2 C\left(1+T^{\theta_{2}}\right) M^{\beta} \leq 1 / 2$. It follows now that $\widetilde{X}$ is stable by $\Phi$ and $\Phi$ is a contraction on $\widetilde{X}$.

Therefore, there exists a unique solution $u \in C\left(I ; H^{s}\right) \cap L^{q}\left(I ; L^{r}\left(|x|^{-r \gamma}\right)\right)$ in the time interval $[0, T]$ with $T=T\left(u_{0}, \alpha, \beta\right)$. Here we cannot take $T=\infty$ even if $\left\|u_{0}\right\|_{H^{s}}$ is small enough to guarantee (5.6), because $\theta_{2}=s / 2>0$. The continuous dependence on the data follows clearly in the same way as before.

\section{REFERENCES}

[1] M. Ben-Artzi and S. Klainerman, Decay and regularity for the Schrödinger equation, J. Anal. Math. 58 (1992), 25-37.

[2] L. Bergé, Soliton stability versus collapse, Phys. Rev. E, 62 (2000), 3071-3074.

[3] J. Bergh and J. Löfström, Interpolation Spaces, An Introduction, Springer-Berlag, Berlin-New York, 1976.

[4] T. Cazenave, Semilinear Schrödinger equations, Courant Lecture Notes in Mathematics, 10. New York University, Courant Institute of Mathematical Sciences, New York; American Mathematical Society, Providence, RI, 2003. 
[5] M. Christ and A. Kiselev, Maximal functions associated to filtrations, J. Funct. Anal. 179 (2001), 409-425.

[6] T. Cazenave and F. B. Weissler, Some remarks on the nonlinear Schrödinger equation in the critical case, Nonlinear Semigroups, Partial differential equations and attractors (Washington, DC, 1987), Lecture Notes in Math. 1394, Springer, Berlin, 1989, 18-29.

[7] T. Cazenave and F. B. Weissler, The Cauchy problem for the critical nonlinear Schrödinger equation in $H^{s}$, Nonlinear Anal., 14 (1990), 807-836.

[8] V. D. Dinh, Scattering theory in a weighted $L^{2}$ space for a class of the defocusing inhomogeneous nonlinear Schrödinger equation, to appear in Adv. Pure Appl. Math.

[9] L. G. Farah, Global well-posedness and blow-up on the energy space for the inhomogeneous nonlinear Schrödinger equation, J. Evol. Equ. 16 (2016), 193-208.

[10] F. Genoud, An inhomogeneous, $L^{2}$-critical, nonlinear Schrödinger equation, Z. Anal. Anwend. 31 (2012), 283-290.

[11] F. Genoud and C. A. Stuart, Schrödinger equations with a spatially decaying nonlinearity: existence and stability of standing waves, Discrete Contin. Dyn. Syst. 21 (2008), 137-186.

[12] C. M. Guzmán, On well posedness for the inhomongeneous nonlinear Schrödinger equation, Nonlinear Anal. Real World Appl. 37 (2017), 249-286.

[13] J. Ginibre and G. Velo, On a class of nonlinear Schrödinger equations I. The Cauchy problem, general case, J. Funct. Anal. 32 (1979), 1-32.

[14] J. Ginibre and G. Velo, The global Cauchy problem for the nonlinear Schrödinger equation revisited, Ann. Inst. H. Poincaré Anal. Non Linéaire, 2 (1985), 309-327.

[15] J. Holmer, S. Roudenko, A sharp condition for scattering of the radial $3 D$ cubic nonlinear Schrödinger equation, Comm. Math. Phys. 282 (2008), 435-467.

[16] T. Kato, On nonlinear Schrödinger equations, Ann. Inst. H. Poincaré Phys. Théor. 46 (1987), 113-129.

[17] T. Kato and K. Yajima, Some examples of smooth operators and the associated smoothing effect, Rev. Math. Phys. 1 (1989), 481-496.

[18] M. Keel and T. Tao, Endpoint Strichartz estimates, Amer. J. Math 120 (1998), 955-980.

[19] E. M. Stein and G. Weiss, Fractional Integrals on $n$-Dimensional Euclidean Space, J. Math. Mech. 7 (1958), 503-514.

[20] R. S. Strichartz, Restrictions of Fourier transforms to quadratic surfaces and decay of solutions of wave equations, Duke Math. J. 44 (1977), 705-714.

[21] M. Sugimoto, Global smoothing properties of generalized Schrödinger equations, J. Anal. Math. 76 (1998), 191-204.

[22] I. Towers and B. A. Malomed, Stable (2+1)-dimensional solitons in a layered medium with sign-alternating Kerr nonlinearity, J. Opt. Soc. Amer. B Opt. Phys. 19 (2002), 537-543.

[23] Y. Tsutsumi, $L^{2}$-solutions for nonlinear Schrödinger equations and nonlinear groups, Funkcial. Ekvac. 30 (1987), 115-125.

[24] M. C. Vilela, Regularity of solutions to the free Schrödinger equation with radial initial data, Illinois J. Math. 45 (2001), 361-370.

[25] K. Watanabe, Smooth perturbations of the selfadjoint operator $|\Delta|^{\alpha / 2}$, Tokyo J. Math. 14 (1991), 239-250.

[26] M. Weinstein, Nonlinear Schrödinger equations and sharp interpolation estimates, Comm. Math. Phys. 87 (1983), 567-576.

Department of Mathematics, Sungkyunkwan University, Suwon 16419, Republic of KoREA

Email address: kimjk809@skku.edu

Email address: yjglee@skku.edu

Email address: ihseo@skku.edu 